\title{
OA05-0 I. In vivo electroporation enhances the immunogenicity of ADVAX, a DNA-based HIV-I vaccine candidate, in healthy volunteers
}

S Vasan*1, A Hurley ${ }^{1}$, SJ Schlesinger ${ }^{1}$, D Hannaman ${ }^{2}$, DF Gardiner ${ }^{1}$, DP Dugin'1, MM Boente-Carrera1 ${ }^{1}$ RM Vittorino1, M Caskey ${ }^{1}$, J Andersen ${ }^{1}$, Y Huang1, J Cox ${ }^{3}$, T Tarragona ${ }^{3}$, DK Gill'3, H Cheeseman ${ }^{3}$, L Clark ${ }^{3}$, L Dally ${ }^{4}$, C Smith ${ }^{4}$, C Schmidt ${ }^{3}$, H Park ${ }^{3}$, E Sayeed ${ }^{3}$, J Gilmour ${ }^{3}$, P Fast ${ }^{3}$, R Bernard ${ }^{2}$ and $\mathrm{DD} \mathrm{Ho}{ }^{1}$

Address: ${ }^{1}$ Aaron Diamond AIDS Research Center, Rockefeller University, New York, NY, USA, ${ }^{2}$ ICHOR Medical Systems, San Diego, CA, USA, ${ }^{3}$ International AIDS Vaccine Initiative, New York, NY, USA and ${ }^{4}$ The EMMES Corporation, Rockville, MD, USA

* Corresponding author

from AIDS Vaccine 2009

Paris, France. 19-22 October 2009

Published: 22 October 2009

Retrovirology 2009, 6(Suppl 3):O3I doi:|0.I|86/I742-4690-6-S3-O3।

This abstract is available from: http://www.retrovirology.com/content/6/S3/O3I

(c) 2009 Vasan et al; licensee BioMed Central Ltd.

\section{Background}

In healthy volunteers, we sought to determine the safety, tolerability, and immunogenicity of ADVAX, a subtype B'/ C, DNA-based, multigenic, HIV-1 vaccine candidate, when injected intramuscularly immediately followed by in vivo electroporation (EP) using the TriGrid ${ }^{\mathrm{TM}}$ Delivery System.

\section{Methods}

Forty healthy volunteers aged 18-60 were enrolled in a double blind randomized phase-I trial. Eight volunteers each received either low dose (LD, $0.2 \mathrm{mg}$ ); mid dose (MD, $1.0 \mathrm{mg}$ ); or high dose (HD, $4.0 \mathrm{mg}$ ) ADVAX or saline placebo via EP. Another eight volunteers received $4.0 \mathrm{mg}$ ADVAX intramuscularly (IM). Vaccinations were given at weeks 0 and 8 . The protocol was subsequently amended to administer a third dose of HD EP/placebo at week 36 to volunteers receiving either HD ADVAX via EP $(n=8)$ or placebo via EP $(n=3)$. Total study follow-up is 14 months.

\section{Results}

There have been no vaccine or device related serious adverse events to date. After two vaccinations in all subjects, the IFNg ELISPOT response rates were IM: $1 / 8$ (13\%), LD-EP: $3 / 8$ (38\%) MD-EP: $7 / 8$ (88\%) and HD-EP: $6 / 8(75 \%)$. In the same order, the mean (range) response to peptide pools spanning all antigens was 72, 120 (70193), 151 (53-440), and 141 (59-336) SFC/million PBMCs. The breadth of the response improved with EP and increasing dosage, with responses to 1, 1, 3, and 4 of the 4 ADVAX gene products. ICCS analysis of ELISPOT responders revealed a balanced CD4+/CD8+ response. There were no responses to placebo, by definition. An analysis of responses after the third vaccination in the high dosage group is ongoing.

\section{Conclusion}

This study is the first demonstration in healthy volunteers that EP in vivo is safe, tolerable, and effective in improving the magnitude and breadth of cellular immune responses to a DNA-based vaccine. 